\title{
Automatic Diagnosis of Liver Tumor in Ct Images
}

\author{
T. Dhiliphan Rajkumar, D. Deepa, J. Jeyaranjani
}

\begin{abstract}
Medical Image Segmentation is the important tool for diagnosing tumor and for planning how to do treatment. The intention of this study is to detect tumor from CT liver images. Initially, liver is segmented from abdomen CT images. Then SVM Classification is included to classify the normal and abnormal liver structure. If it is abnormal then the tumor will be segmented from liver structure. This technique is computed using sensitivity, specificity and accuracy and is providing good result.
\end{abstract}

Index Terms - tumor detection, Liver Segmentation, SVM Classification, hepatic tumor

\section{INTRODUCTION}

The liver plays a various roles in the human body and the major role in metabolism. The liver also plays the role in decay of red blood cells, regulation of glycogen storage, hormone creation, plasma protein synthesis, and detoxification. It is a digestive gland which produces bile, which helps for digestion through the emulsification of lipids. Hepatocellular carcinoma is commonly caused in liver cancer. The cancer that begins in the colon and spreads to the liver.

Computerized Tomography (CT) scan uses X-rays and a computer to generate exhaustive images inside the body. CT scans are also sometimes known as CAT scans, which stands for Computerized Axial Tomography. The images produced by a CT scan are called tomograms and are more detailed than standard X-rays. It also can produce images of structures inside the body, including the internal organs, blood vessels, bones and tumors.

The tumor segmentation from CT images gives good detection rate. There exits the similarity between tumor and other tissues. Hence the tumor detection techniques are applied for the images from clinical routine. To detect the tumor in the CT images first the liver is segmented from abdomen using morphological operations. Then SVM classification will classify the liver as normal or abnormal as told already. If the liver is abnormal then tumor will be detected from the liver structure using Knowledge based constraints. In this work, datasets of more patients were processed using computer aided diagnosis. The outcomes were computed using some of the metrics like sensitivity, specificity and accuracy by comparing it with respect to normal segmentation carried out by an expert radiologist.

Revised Manuscript Received on December 15, 2019

* Correspondence Author

Dr. T. Dhiliphan Rajkumar*, Assistant Professor,Department of and Education,Anand Nagar, KrishnanKoil .

Mrs. D. Deepa , Assistant Professor, Department of Computer Science Kongu Engineering College

Mrs. J. Jeyaranjani, Assistant Professor,Department of computer Science and Engineering,Kalasalingam Academy of Research and Education,Anand Nagar, KrishnanKoil. computer Science and Engineering,Kalasalingam Academy of Research

\section{A. Motivation And Justification}

Park et al. [14] proposed a method in which they applied intensity histogram transformation for segmentation of the liver and a posteriori classification leads to a binary mask. Once the morphological processing is applied to the mask, the tumors are identified within the mask area. K.-S. Seo.[6], in his method it segments the liver first, then by using the optimal threshold value hepatic tumor is segmented with minimum total probability error. This approach produces diverse false positives, but promising results are obtained.

Ciecholewski et al [10], discussed about a method in which segmented the liver using the contour model, and then enhance the image using histrogam transformation. Region growing algorithm using intensity distribution is proposed by Zhao et al. [17]. In Arakeri et al. [1], seed points are selected automatically using modified region growing algorithm and also it proposed a method to find the threshold value that incorporates fuzzy c-means clustering algorithm. Massoptier and Casciaro.[12] proposed that, the liver is segmented by the approach statistical method then the tumor is classified by analyzing the wavelet. Chen and Metaxas [3], had used Markov Random Field (MRF) estimation and also Deformable models to segment the tumors.

Shang et al. [16] proposed a model in which active contour with an embedded classifier using a Gaussian mixture model build in to the intensity distribution of the medical images for segmenting liver, vessels and lesions.

Lu et al. [9], worked to obtain the active contour in the tumor boundary manually. Segmentation based on Watershed technique [5] in this, edge detection, the watershed algorithm and region merging approaches are used. In Knowledge-Based Constraints[13], the gray level intensity contrast was enhanced; then image was added to itself, to isolate tumour directly, finally the image is segmented by threshold based method; Since it is sensitive to noise, the morphological filter is applied as the postprocessing.

Marius George Linguraru.[11], in his work statistic adaptive threshold initialization is done. The user needs to select seed point with the label and background. Then the image is represented in an undirected weighted graph. Every node of image represents each pixel. The segmentation is obtained in graph cut. Every region indicates a sub graph. Sergio Casciaro[15] proposed a method in which the tumor is separated first by thresholding method.

The main problem in tumour detection techniques are computational time, false positive rate, initial assumption. Motivated by this concept, an attempt is taken to implement the liver tumour segmentation by overcoming these disadvantages. The different approaches for liver tumour detection have been developed and Knowledge based constraints $(\mathrm{KBC})$ is found to be one of the best method. The detection of tumour using CT image give good results compared to MRI scan images. Justified by this, it is essential to implement a novel method for the tumour 


\section{Automatic Diagnosis of Liver Tumor in Ct Images}

detection techniques by combining KBC method with SVM classifier.

\section{B. Outline of the Proposed Work}

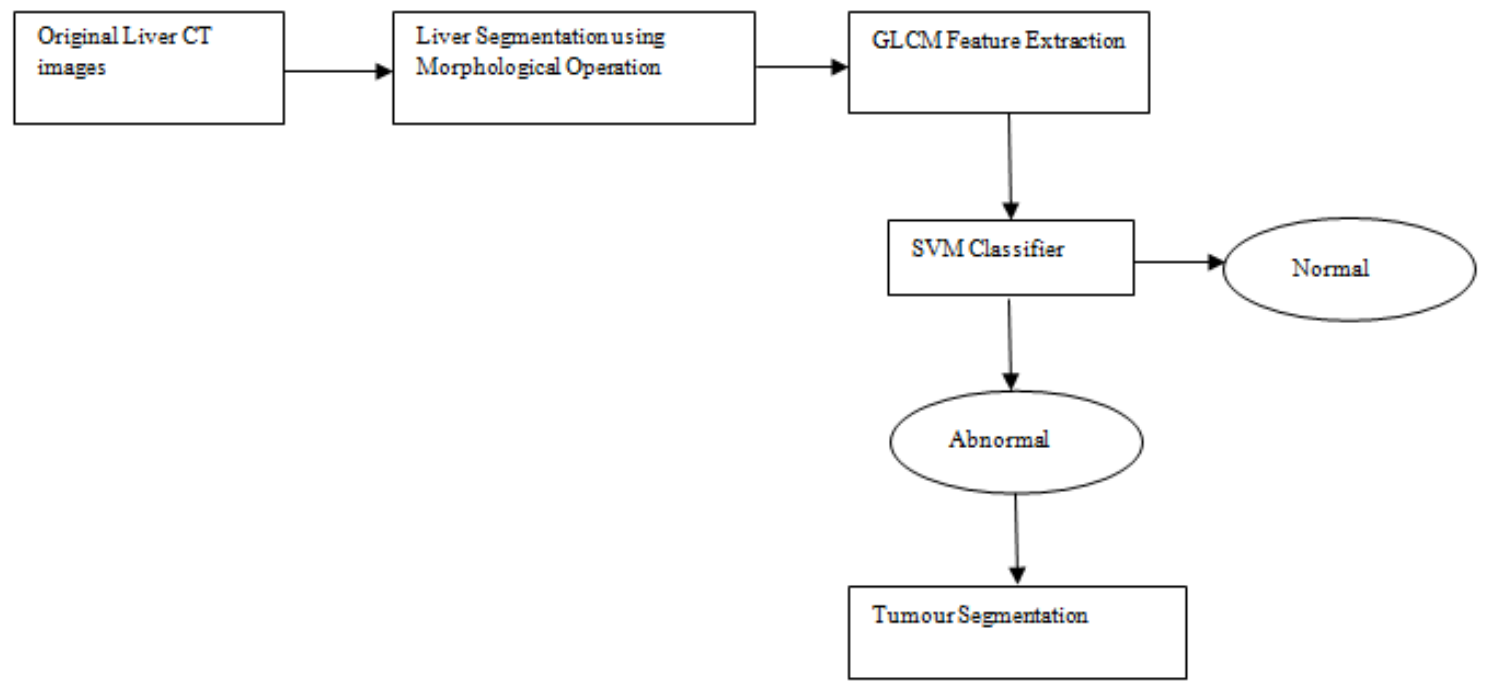

Fig 1 A novel system for Automatic Computer Aided tumour detection techniques

Fig.1. is the overall working model for tumour detection techniques. The input will be the original liver CT image. The liver undergo segmentation by the use of morphological operations. Then the features are extracted from the segmented liver structure using feature extraction method called Gray Level Co-occurrence Matrix (GLCM). Then these features are given as the input for Support Vector Machine (SVM) classifier for checking whether the liver as normal or abnormal. If the tumour is abnormal then it undergoes tumour segmentation process.

In Tumour Segmentation technique Knowledge based constraint is addressed first, in which linear contrast Stretching and morphological operations are used for segmenting the tumour. Finally, performance is evaluated using sensitivity, specificity and accuracy.

\section{Organization Of The Paper}

Section II Automatic computer aided liver tumor detection technique is discussed. And section III is the experimental result and performance analysis. Finally, section IV is the conclusion and the future scope.

\section{TUMOR DETECTION METHOD}

\section{A. Liver Segmentation}

Segmentation of liver is carried out in CT abdomen images. First the neighborhood pixels are calculated with the structuring elements. Here $N H O O D=$ getnhood(SE), which provides the neighborhood linked with the structuring element SE. Then, range filter is useful to find the output pixel with the range value = max.value - min.value of the 3-by-3 neighborhood around the equivalent pixel in the input image. Maximum value and minimum value in the specific neighborhood is determined by the use of morphological functions imdilate and imerode. The input image is similar to dimension. The output image is same as of the input image. NHOOD is a multidimensional array of 0 's and 1 's. In NHOOD's, the size should be odd in every dimension. Here the Range filter is to predict the center element of the neighborhood using the formula $((\operatorname{size}(N H O O D)+1) / 2)$. Fig. 2 is the input i.e. raw CT image and Fig 3 is the output after getting neighborhood with structuring element and applying range filter.

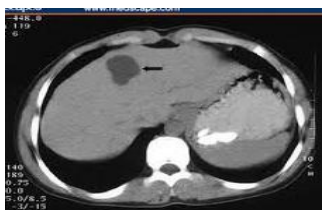

Fig 2 Raw CT image

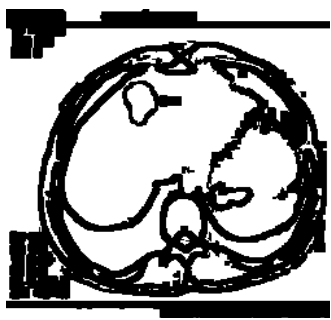

Fig 3 Image after getting neighborhood and range filter 
Finally, Morphological operation is applied to segment the liver structure. Area opening operation avoids a binary image of every connected component (objects) which has lesser than $\mathrm{P}$ pixels, generating other binary image, BW2. This procedure is called as area opening. The border is cleared for the output image because it is lighter than the input image. The input image IM and the output image IM2 can be a grayscale or binary image correspondingly. The standard connectivity is 8 and 26 for 2 and 3 dimensions respectively.

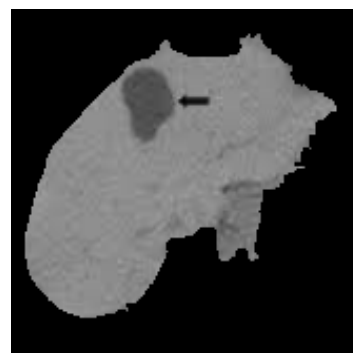

Fig 4 Segmented liver from CT image

\section{B. Feature extraction}

Feature extraction is needed for SVM classifier in this the input images size is very big and also lot of them are redundant. So it is very important to reduce the size into a collection of features, for that purpose ROI feature extraction by the use of GLCM technique. The statistical information about the image is needed for feature extraction [6].

These are some of the statistical information

1. Area: It provides the real number of pixels in ROI.

2. Convex Area: It provides the pixel count in convex image of the ROI.

3. Equivalent Diameter: It is the diameter of the circle. It is calculated using the formula,

Equivalent Diameter $=\frac{\sqrt{4 * \text { Area }}}{\sqrt{\pi}}$

4. Solidity: It is proportionally equal to pixels in the convex hull. It is evaluated using,

$$
\text { Solidity }=\frac{\text { Area }}{\text { Convex Area }}
$$

5. Energy: It is the total of squared components in the GLCM and the value lies in the range of $[0,1]$.

$$
\text { Energy }=\sum_{k=0}^{n} p^{2}(i, j)
$$

6. Contrast: It is to determine the contrast between the power of pixel and adjacent pixels over the entire ROI. The formula for contrast is shown below

7. Contrast $=\sum_{i}^{N} \sum_{j}^{N}(i-j)^{2} p(i, j)$

(4)

where $\mathrm{N}$ is the number of various gray levels.
8. Homogeneity: It is to find the similarity of the distribution of elements in GLCM of every ROI and the value lies in the range of $[0,1]$.

$$
\text { Homogeneity }=\sum_{i, j} \frac{p(i, j)}{1+|i-j|}
$$

9. Correlation: It is to evaluate the correlation of pixel to its neighbor over the ROI.

$$
\text { Correlation }=\sum_{i}^{N} \sum_{j}^{N} \frac{p(i, j)-\mu_{r} \mu_{c}}{\sigma_{r} \sigma_{c}}
$$

10. Eccentricity: It is the ratio of distance from focus of the ellipse and major axis length.

GLCM comprises data concerning the positions of pixels with identical gray level values. Here, co-occurrence matrix is a 2-dimensional array $\mathrm{P}$ where rows and columns indicate a collection of probable image value. Here, feature extraction from the CT liver image takes place in four directions $\left(0^{\circ}, 45^{\circ}, 90^{\circ}\right.$ and $\left.135^{\circ}\right)$. These directions are used for finding the co-occurrence matrix. Statistical features are extracted from all there four direction.

\section{Classification using SVM}

Computers have capability to gain knowledge of without being explicitly programmed; this field of study is Machine Learning. This learning algorithm then outputs a function known as hypothesis. The rule or set defines the hypothesis is, what are "learned" from the training set. The feature set has been calculated for every pixel can be employed by SVM classifier to check whether every pixel has a tumor or normal pixel. The classifier used is a binary classifier. It provides the result like normal or abnormal. So in our work with normal liver tumor images abnormal tumor image have to be included. Then only it is easy for us to achieve the desired results. By supervised learning, we can classify various diseases in liver lesion. The process has two phases namely a training phase and testing phase. At the first phase, the input is the pixel features and its respective manual labels, and the output is a model which utilizes the features to predict the equivalent label. This phase is carried out only once, because the model can be used to classify new data. In the testing phase, the input is already known model and pixel features with no respective classes, and the output of the testing phase is the predicted one for the pixels depending upon their features. The main objective of the SVM classifier is to classify data that have similar feature values into groups ie. (normal or abnormal).

\section{Tumor Segmentation}

If the SVM classifier classifies the liver structure as abnormal then tumour segmentation process is done. The similar gray level liver parenchyma and tumor tissues are obtained by improving the contrast of the segmented slice. In tumor segmentation, linear contrast stretching is employed for increasing the variation among liver tissue and tumors. Formula applied to this performance is given in Eq. (1):

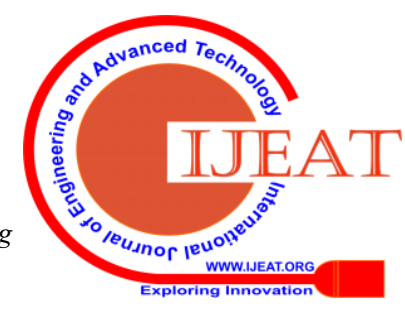




$$
I^{\prime}=\frac{I_{\max }^{\prime}-I_{\min }^{\prime}}{I_{\max }-I_{\min }}\left(I-I_{\min }\right)+I_{\text {min }}^{\prime}
$$

where, $\mathrm{I}$ is gray level before transformation and $\mathrm{I}^{\prime}$ is gray levels after transformation. I'max is the highest gray level after transformation and I'min is the lowest gray levels after transformation, Imax is the maximum gray level and Imin is the minimum gray level in the liver region prior to transformation. Finally, liver and tumor is differentiated. Formula(2), to add the improvement to the same image.

$$
R(i, j)=I^{\prime}(i, j)+I^{\prime}(i, j)
$$

where $R(i, j)$ is the image after applying this it will reach 255 and will appear as white. We obtain the pixels of tumor tissues dark. The outcome of addition is image background as well liver tissue which appear in white background with some pepper noise, and tumors which appeared as dark spots with range of gray levels. In order to remove the noise and make the region of tumor more homogeneous as in shown Fig. 3(a), Gaussian smoothing is used as in (3), where $x$ is the distance from the origin in the horizontal axis, $y$ is the distance from the origin in the vertical axis, and $\sigma$ is the standard deviation of the Gaussian distribution.

$$
G(x, y)=\frac{1}{2 \pi \sigma^{2}} e^{-\frac{x^{2}+y^{2}}{2 \sigma^{2}}}
$$

Next step is that iso-data thresholding operation is done to turn the image into binary where the tumor region is appeared as black blob on white space, followed by morphological holes filling operation like erosion and dilation as in Fig 5.

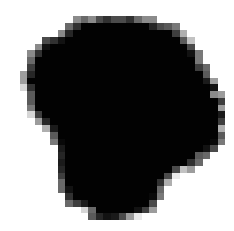

Fig 5 Final Segmented tumour

\section{EXPERIMENTAL RESULTS AND PERFORMANCE ANALYSIS}

In this, liver is segmented from the CT images. Next, the features are extracted from the liver. These features are provided as the input for SVM classifier for classifying whether the liver is normal or abnormal. If the liver is abnormal then it undergoes tumour segmentation process. If the liver is normal then there will be no need of tumour segmentation. In which tumour is segmented using Knowledge Based Constraints methods. The result is the segmented tumour in CT image, but the detected result may vary according to the algorithm's sensitivity, specificity and accuracy. If the specificity is high then, the tumour detection algorithm gives the result correctly. While checking the percentage of accuracy, if the percentage is high it will give the exact detection of liver tumour. Since this whole paper concentrated only on Liver CT the best algorithm can be chosen according to how much tumour is detected correctly.

\section{A. Experimental result}

This experiment detects the tumour in CT image. The segmented tumour from the CT scan image is displayed in fig 6. Here, if the SVM classifier classifies the liver structure as tumour will be segmented the as tumour.

Table 1 Sensitivity, Specificity and accuracy of various tumour detection techniques in percentage $(\%)$

\begin{tabular}{|c|c|c|c|}
\hline Image & Sensitivity(\%) & Specificity(\%) & Accuracy(\%) \\
\hline 1 & 98 & 98 & 98 \\
\hline 2 & 98 & 99 & 98 \\
\hline 3 & 99 & 98 & 99 \\
\hline 4 & 98 & 99 & 98 \\
\hline 5 & 98 & 98 & 98 \\
\hline
\end{tabular}

\section{B. Performance Evaluation}

For performance evaluation some of the metrics used are sensitivity, specificity and accuracy. The formulas to calculate them are listed below.

$$
\begin{gathered}
\text { Sensitivity }=\frac{\mathrm{TP}}{\mathrm{TP}+\mathrm{FN}} \\
\text { Specificity }=\frac{\mathrm{TN}}{\mathrm{TN}+\mathrm{FP}} \\
\text { Accuracy }=\frac{T P+T N}{\mathrm{TP}+\mathrm{FP}+\mathrm{TN}+\mathrm{FN}}
\end{gathered}
$$

where TP is number of true positive, $\mathrm{FN}$ is number of false negative, TN is number of true negative and FP is number of false positive.

\begin{tabular}{|c|c|c|}
\hline Test Images & $\begin{array}{c}\text { Liver } \\
\text { Segmentation }\end{array}$ & $\begin{array}{c}\text { Tumour } \\
\text { Segmentation }\end{array}$ \\
\hline & & \\
\hline & & \\
\hline & & \\
\hline
\end{tabular}

Blue Eyes Intelligence Engineering \& Sciences Publication 


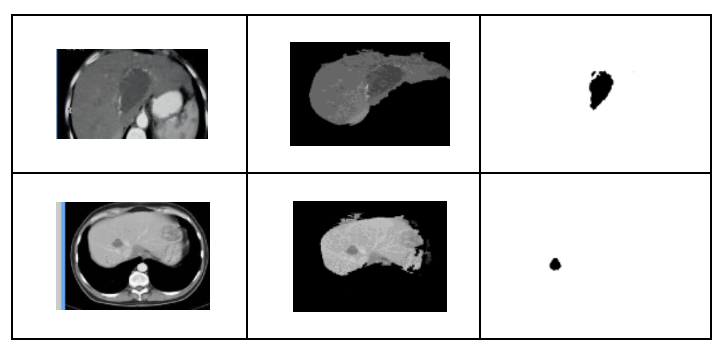

\section{Fig 6 Segmented Liver from CT image of different patients}

Table 1 present the sensitivity, specificity and accuracy for segmented liver tumour image with manually segmented liver tumour images.

Here 5 test images are considered and sensitivity, specificity and accuracy of each image is identified for each procedure. It is observed that from the table the proposed work is providing better results

\section{CONCLUSION AND FUTURE SCOPE}

In this work, segmentation and classification of liver tumor from the abdomen CT image is proposed. It assists the doctors for predicting and analyzing liver tumor and analysis for tumor surgery. Once pre-processing is done, the liver is segmented and classified whether it is normal or abnormal. This technique shows improvement significantly. Experimental results show that the proposed work achieves $98.2 \%$ of sensitivity, $98.4 \%$ of specificity and $98.2 \%$ of overall accuracy. This paper helps to obtain almost the tumour detected area correctly. Further researches also strive towards improving accuracy, precision, computational speed, automation and reduction of manual interaction.

\section{REFERENCES}

1. Arakeri.M.P and Lakshmana. (2010) "Automatic segmentation of liver tumor on computed tomography images," in Proceedings of the International Conference and Workshop on Emerging Trends in Technology. Mumbai, Maharashtra, India: ACM.

2. Chen.L and Metaxas.D.(2005) "A hybrid framework for 3D medical image segmentation," Journal of Medical Image Analysis, vol. 9, pp. 547-565.

3. Haralick R. M., Shanmugam K., and Dinstein .I, "Textural features forimage classification,” IEEE Trans. Syst., Man Cybern., vol. 3, no.6,pp. 610-621, Nov. 1973

4. Jianhua.L, Zhongyi.W, and Rui.Z.(2009) "Liver Cancer CT Image Segmentation Methods Based on Watershed Algorithm," in International Conference on Computational Intelligence and Software Engineering (CiSE '09). Wuhan, China, pp. 1-4.

5. Kyung-Sik Seo and Tae-Woong Chung. (2005). 'Automatic Boundary Tumor Segmentation of a Liver', In ICCSA 2005, pages 836-842.

6. Lu.R, Marziliano.P, and Hua Thng.C. (2005) "Liver Tumor Volume Estimation By Semi-Automatic Segmentation Method," presented at 27th Annual International Conference of the Engineering in Medicine and Biology Society (EMBS '05), Shanghai, China.

7. Marcin Ciecholewski and Marek R. Ogiela. (2007) 'Automatic Segmentation of Single and Multiple Neoplastic Hepatic Lesions in CT Images', In IWINAC '07: Proceedings of the 2nd international work-conference on Nature Inspired Problem-Solving Methods in Knowledge Engineering, pages 63-71.

8. Marius George Linguraru*, William J. Richbourg, Jianfei Liu, Jeremy M. Watt, Vivek Pamulapati,Shijun Wang, and Ronald M. Summers.(2012)'Tumor Burden Analysis on Computed Tomography by Automated Liver and Tumor Segmentation' in Medical Imaging, IEEE Transactions on (Volume:31, Issue: 10 ) Page(s):1965 - 1976.
9. Massoptier.L and Casciaro.S. (2008) "A New Fully Automatic and Robust Algorithm for Fast Segmentation of Liver Tissue and Tumors from CT Scans," Journal of European Radiology, vol. 18, pp. 1658-65.

10. Nader H. Abdel-massieh, Mohiy M. Hadhoud, and Khalid M. Amin (2010)'Automatic Liver Tumor Segmentation from CT

Scans with Knowledge-based Constraints', in Biomedical Engineering Conference (CIBEC), 2010 5th Cairo International Page(s): $215-218$.

11. Seung-Jin Park, Kyung-Sik Seo and Jong-An Park. (2005) 'Automatic Hepatic Tumor Segmentation Using Statistical Optimal Threshold' In ICCS, pages 934-940.

12. Sergio Casciaro, Roberto Franchini, Laurent Massoptier, Ernesto Casciaro, Francesco Conversano,Antonio Malvasi, and Aimè LayEkuakille.(2012)' Fully Automatic Segmentations of Liver and Hepatic Tumors From 3-D Computed Tomography Abdominal Images: Comparative Evaluation of Two Automatic Methods',in Sensors Journal, IEEE (Volume:12, Issue: 3 ) Page(s):464 - 473.

13. Shang.Y, Markova.A, Deklerck.R, Nyssen.E, Yang.X, and de Mey.J.(2010) "Liver segmentation by an active contour model with embedded Gaussian mixture model based classifiers," in Optics, Photonics, and Digital Technologies for Multimedia Applications, vol. 7723, P. Schelkens, T. Ebrahimi, G. Cristobal, F. Truchetet, and P. Saarikko, Eds. Brussels, Belgium: SPIE, pp. 772313-7.

14. Zhao.B, Schwartz.L.H, Jiang.L, Colville.J, Moskowitz.C, Wang.L, Leftowitz.R, Liu.F, and Kalaigian.J, (2006) "ShapeConstraint Region Growing for Delineation of Hepatic Metastases on Contrast-Enhanced Computed Tomography Scans," Journal of Investigative Radiology, vol.41, pp. 753-762.

\section{AUTHORS PROFILE}

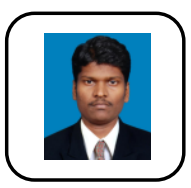

T. Dhiliphan Rajkumar completed his B.E (2009) Computer Science and Engineering in Arulmigu Kalasalingam College of Engineering, Anna University. $\mathrm{He}$ received M.E (2011) Computer Science and Engineering in Muthayammal Engineering College, Anna University. He pursed his Ph.D (2017) degree in Computer Science and Engineering, Manonmaniam Sundaranar University, India. He has a strong passion in Web Mining, Pattern recognition and Social networking. He is currently working as Assistant Professor in Kalasalingam Academy of Research and Education.

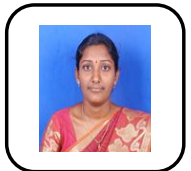

D. Deepa completed her B.E (2009) Computer Science and Engineering in Velalar College of Engineering and Technology, Anna University. She received M.E (2011) Computer Science and Engineering in Muthayammal Engineering College, Anna University. She has a strong passion in Cloud Computing, Fog Computing and Web Mining. She is currently working as Assistant Professor in Kongu Engineering College, Erode.

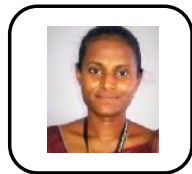

Mrs. J. Jeyaranjani completed her B.E(2009) Computer Science and Engineering at KLN College of Information Technology. She received her M.Tech (2011) Computer Science and Engineering at Veltech University. She is pursuing her Ph.D degree in Computer Science and Engineering, Kalasalingam Academy of Research and Education. Her research interest is Smart Grid, Data Analytics, Machine Learning, and IoT. Currently, she is working as Assistant Professor in the Department of CSE, Kalasalingam Academy of Research and Education, India. 